\title{
A Probabilistic based Redundancy Control Algorithm for Wireless Sensor Network
}

\author{
Haitao Wang, Jianzhou Li, Li Yan \\ College of Communications Engineering, PLA Univ. of Sci. \& \\ Tech. \\ Nanjing 210007, China
}

\author{
Xueping Zhang, Hui Chen \\ Information Management Center, PLA Univ. of Sci. \& \\ Tech. \\ Nanjing 210007, China
}

\begin{abstract}
Existing redundancy control algorithms in WSN often have some evident shortcomings such as hypothesis is too much, application backgrounds are not clear, the precision of redundancy control is low and computing complexity is high. In this paper a probabilistic based redundancy control algorithm (PBRCA) is proposed. Aiming at specific application scenarios, PBRCA introduces the probabilistic estimation theory, taking uncertainty in reasonable range as cost to determine redundant nodes in network and allow as many redundant nodes as possible to sleep. This algorithm requires fewer assumed conditions and less computational complexity as well as high flexibility. In addition, it can adapt to various redundancy control requirements of WSN with different network scales and node densities. Simulation results show that, PBRCA can control network topology node redundancy effectively with little cost and improve network efficiency.
\end{abstract}

Keywords-Wireless Sensor Network; Redundancy control; Probabilistic Estimation; Independency Set; Overlap Set

\section{INTRODUCTION}

Deployment environment of Wireless sensor network (WSN) is often harsh, such as battleground and disaster relief environment, where nodes are usually spread randomly. Since it is difficult to charge nodes in WSN and the size of node is very constrained, energy saving is always a hot topic in WSN [1]. The monitoring area of a sensor node is usually a circular region $\mathrm{s}$ and its radius is referred to as sensing radius or covering radius. In Boolean coverage model, the situation within circle region can be perceived by the node [2]. For simplicity, Boolean coverage model is adopted in this paper and common domain coverage is used in particularly [3]. Node's communication range is usually adjustable an, nodes' communication range is also a circular region. Thus the communication distance of a node is often referred to as communication radius which is often greater than or equal to its coverage radius.

Coverage of WSN refers to the range and proportion of the specified region monitored by sensor nodes and connectivity refers to the ratio of nodes in WSN which can communicate directly or indirectly. Coverage determines the monitoring ability of WSN and connectivity is relevant to reliable end to end transmission of monitored data [4]. If coverage and connectivity of the network are not affected when some nodes go to sleep then these nodes are referred to as redundant nodes. One important aim of redundancy control algorithm in WSN is to make redundant nodes sleep to reduce data conflicts and save energy, and thus prolonging the network lifetime. Most redundancy control algorithms require many preconditions and have higher computation complexity. Dynamic transmission power control algorithm detects the number of neighbors of each node and then adjusts the transmission power so that the number of neighbor nodes can be kept in a reasonable range. But this algorithm cannot control network coverage redundancy [5]. Coverage configuration protocol (CCP) can configure WSN with arbitrary coverage degree and full connectivity. However, when the communication range is less than twice of coverage range, CCP requires combining with SPAN algorithm and its computing complexity is $\mathrm{O}\left(d^{3}\right)$ with $\mathrm{d}$ being the number of nodes in the maximum perception field. Area Dominating Set (ADS) protocol can construct a network with full coverage and connectivity, but it assumes that the communication distance is equal to sensing distance and the algorithm is s centralized algorithm, whose computation complexity is also $\mathrm{O}\left(d^{3}\right)$ which is unsuitable for large-scale distributed WSN. Optimized Geometrical Density Control (OGDC) algorithm also can configure a network with full coverage and connectivity and has high energy efficiency. But in the different situations this algorithm behaves quite differently and the execution time is longer.

In this paper a probabilistic based redundancy control algorithm (PBRCA) is proposed. Aiming at specific application scenarios, PBTCA introduces the probabilistic estimation theory, taking uncertainty in reasonable range as cost to determine redundant nodes in network and allow as many redundant nodes as possible to sleep. This algorithm requires fewer assumed conditions and less computational complexity as well as high flexibility.

\section{REDUNDANCY CONTROL ALGORITHM BASED ON PROBABILITY ESTIMATION}

\section{A. Coverage redundant nodes}

WSN deployment environment is often special and it is very difficult to completely cover the monitoring area. Wireless sensor nodes monitor certain features and changes of objects in environment, which may be physical, chemical or biological characteristics. The features of actual objects are similar within certain space region, that is to say, data observed at the point A may also be observed in the adjacent region of A. So full network coverage sometimes is unnecessary. This is the theory basis of allowing a certain degree of uncertainty. But in order to ensure the fulfillment of monitoring task of WSN, network coverage ratio should be maintained above a certain level. In fact, the expected value Q of the coverage ratio in WSN can serves as a metric 
of service quality. Meanwhile, the hold time of network coverage can be used as the metric of network lifetime [4].

In fact coverage ranges of randomly dispersed nodes in WSN are often overlapped. If the coverage range of a node is covered by other neighbor nodes with very high proportion of $x \%$, e.g. $x>95$, then forcing this node sleep will not affect network coverage and connectivity basically. Therefore, nodes satisfying above requirement can be regarded as coverage redundant nodes.

\section{B. Algorithm thought}

In WSN many nodes are often spread in random uniform distribution approximately and node density is usually high. So some redundant nodes exist in this network environment. As shown in Figure 1, node A receives signal from node B, and if the distance $\mathrm{D}$ between them can be known, then the overlapped area of the coverage range between node $\mathrm{A}$ and $\mathrm{B}$ can be calculated in formula (1).

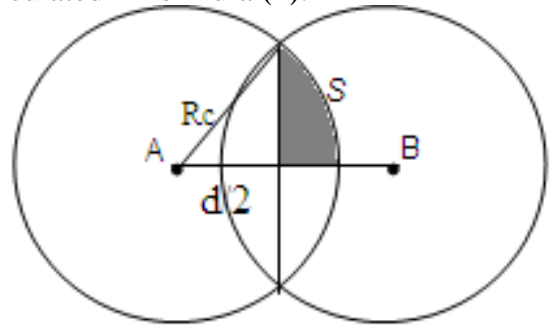

Fig.1 Coverage overlap of two nodes

$$
\mathrm{sc}=4 s=2 * \operatorname{acos}\left(\frac{d}{2} / \mathrm{Rc}\right) * \mathrm{Rc}^{2}-2 * \frac{d}{2} * \operatorname{sqrt}\left(\mathrm{Rc}^{2}-\left(\frac{d}{2}\right)^{2}\right)
$$

$\mathrm{S}_{\mathrm{c}}$ indicates overlapped coverage area of node $\mathrm{A}$ and $\mathrm{B}$ and $\mathrm{d}$ represents the distance between the two nodes, Rc is the coverage radius. The overlapped coverage ratio of node $\mathrm{A}$ is $\mathrm{P}_{\mathrm{AB}}$ :

$$
\mathrm{p}_{\mathrm{AB}}=\mathrm{S} \mathrm{c} / \mathrm{S}
$$

$\mathrm{S}$ is coverage area of $\mathrm{A}$. In the following, $\mathrm{p}_{\mathrm{Ax}}$ represents the ratio that the coverage of node $\mathrm{A}$ is covered by node $\mathrm{X}$. Because nodes obey random uniform distribution, three nodes A, B, C are deployed independent. Therefore the probability of any point a in the coverage of $\mathrm{A}$ not covered by coverage areas of $\mathrm{B}$ and $\mathrm{C}$ meets:

$$
\mathrm{p}_{\text {not_c }}=\left(1-\mathrm{p}_{\mathrm{AB}}\right)\left(1-\mathrm{p}_{\mathrm{AC}}\right)
$$

Whereas the probability that $\mathrm{a}$ is located in the overlapped area of $B$ and $C$ is $p_{c}=1-p_{\text {not }} c$. When node $A$ can receive signals from more nodes, overlapped coverage continues to increase and $\mathrm{p}_{c}$ increases too. When $\mathrm{p}_{c}$ is greater than a certain value Preq ,e.g. Preq $=95 \%$, it is thought that node $\mathrm{A}$ is a redundant node according to above analysis, and A can go to sleep.

\section{Algorithm description}

The flowchart of PBRCA algorithm is depicted in figure 2. At first, each node in WSN broadcasts messages using the same power, and then calculates the distance between nodes, according to the received signal intensity. Next, $\mathrm{p}_{\mathrm{AB}}, \mathrm{p}_{\mathrm{AC}}$,
$\mathrm{p}_{\mathrm{AD}} \cdots$ can be calculated form formula 1 and the value of pc can be calculated according to formula 2 .

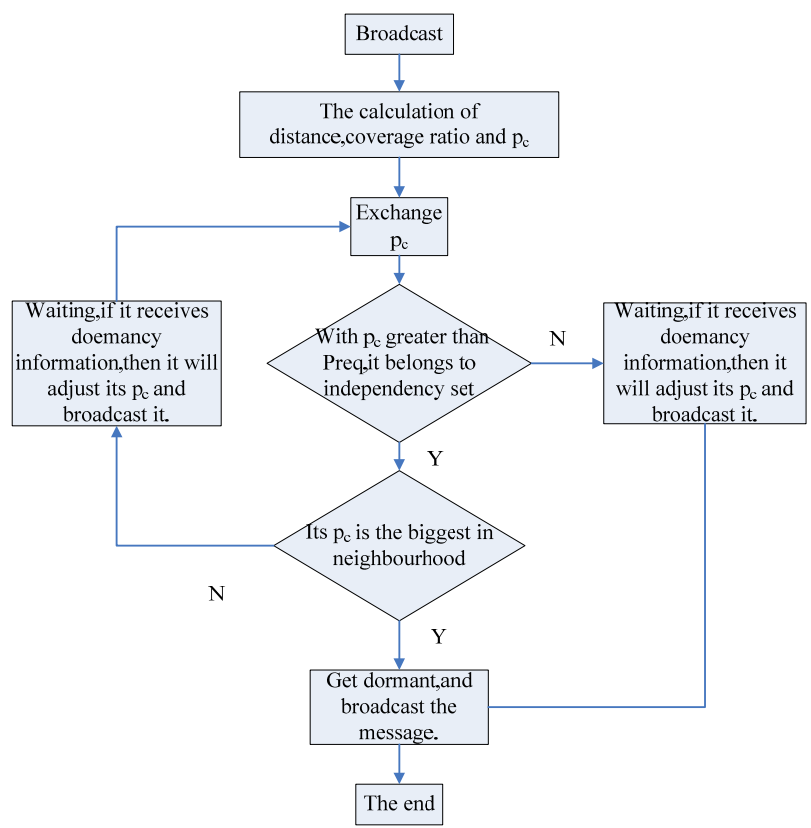

Fig.2 Flowchart of PBRCA algorithm

$\mathrm{p}_{\mathrm{c}}>$ Preq is the essential condition that a node can go to sleep but it is not the sufficient condition. Because nodes that meet this condition in an area may be more than one and they may overlap each other. When a node goes to sleep, its neighbor nodes cannot meet sleep requirements. In order to get better network performance, the node with highest pc should go to sleep first.

After nodes calculate their own pc values, adjacent nodes exchange $\mathrm{p}_{c}$ each other. Then each node will check whether its $\mathrm{p}_{\mathrm{c}}$ is the highest value in neighborhood. If meeting this condition, then it is the most suitable node to sleep. Once a node going to sleep it will inevitably change pc values of neighbor nodes. So nodes getting dormant should broadcast this message and other adjacent ones that receive the message will adjust their values of $\mathrm{p}_{c}$.

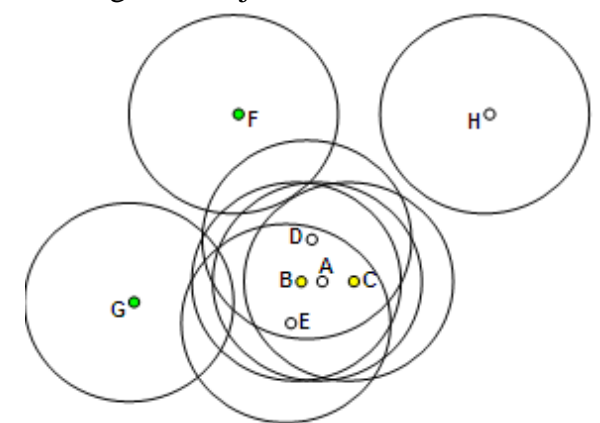

Fig.3 Overlap set, independency set and best dependency

set After adjusting $\mathrm{p}_{c}$, nodes will be in working state if its $\mathrm{p}_{\mathrm{c}}$ is less than Preq, else it can still go to sleep. But 
dormancy of neighbor nodes is correlated. In this paper concepts of coverage overlap set, independency set and best dependency set of any node A are given as follows.

Overlap set: set of all nodes that have overlapped coverage with node A.

Independency set: set of nodes which satisfy the condition that all nodes in this set have overlap coverage with node $\mathrm{A}$ and even excluding this set node A still can go to sleep.

Best dependency set: set of the minimum number of nodes that make node A meeting dormancy condition in WSN.

As shown in Figure 3, overlap set of node A is $\{B, C, D$ $E, F, G\}$ and $\{D, E, F, G\}$ forms an independency set of node $A$ and $\{B, C\}$ is the best dependency set of node $A$.

The independency sets of node A could be more than one. In order to make more nodes get dormant, the maximum independency set of A should be selected allowing more nodes in the set have chance to get dormant. Overlap set minus the best dependency set i.e. the complementary set of the best dependency set is the most ideal dependency set. The best dependency set can be calculated through picking out the minimum combination of nodes from the overlap set of node A whose $\mathrm{p}_{\mathrm{c}}$ is greater than Preq. But the computation complexity of the best dependency set is greater than $\mathrm{O}\left(2^{d}\right)$, where $d$ represents the number of nodes in the maximal overlap set degree. Obviously this computation complexity is too high. For lowing computation complexity the relatively optimal independency set node A can be achieved. Considering the actual situation, dormant nodes satisfy the dormancy condition mainly due to the coverage of adjacent nodes, the relatively distant nodes contribute little. So in PBRCA algorithm relatively near nodes are checked first and a node with a minimum value of $1-\mathrm{p}_{\mathrm{ax}}$ is selected and added to set $G$ (initially $G$ is empty). The product of elements in $G$ is calculate and if the product is greater than 1-Preq, then it continues selecting the node with second smallest value of 1- $\mathrm{p}_{\mathrm{Ax}}$ and adds it to set $\mathrm{G}$, repeating above procedure until the product is less than1-Preq (node A meets the dormancy condition). The complementary set of $\mathrm{G}$ is relatively optimal independency set node A as shown in figure 2. According to above procedure, the complexity for the calculating relatively optimal independency set is $\mathrm{O}\left(d^{2}\right)$.

When node $B$ receives the dormancy information of node $\mathrm{A}$, it adjusts its own $\mathrm{P}_{\mathrm{c}}$ and checks whether it belongs to independency sets of node $\mathrm{A}$. If meeting above condition and $\mathrm{p}_{\mathrm{c}}>$ Preq , then node $\mathrm{B}$ repeats the process of selecting dormant nodes. Otherwise it will be no longer qualified to be dormant and enters working state. When all nodes have determined their own states, the algorithm ends.

\section{SIMULATION TEST AND ANALYSIS}

\section{A. Experimental environment}

OMNet4.0 is used as the simulation tool and free space model is employed. Parameter configuration in the test is shown in table 1.
TABLE 1 PARAMETER CONFIGURATION

\begin{tabular}{|c|c|}
\hline Net coverage's area & $500 * 500 \mathrm{~m}^{2}$ \\
\hline Node communication & $100 \mathrm{~m}$ \\
\hline Node coverage radius & $50 \mathrm{~m}$ \\
\hline The number of nodes & adjustable \\
\hline Preq & adjustable \\
\hline
\end{tabular}

\section{B. Simulation results}

To evaluate the efficiency of PBRCA algorithm, dormancy ratio, original coverage ratio and new coverage ratio are used as evaluation metrics. According to the relationship between service quality and the number of nodes [7], the desired number of nodes that meets Preq $=99 \%$ is 145 , and considering the edge effect, node number is set to 160 .

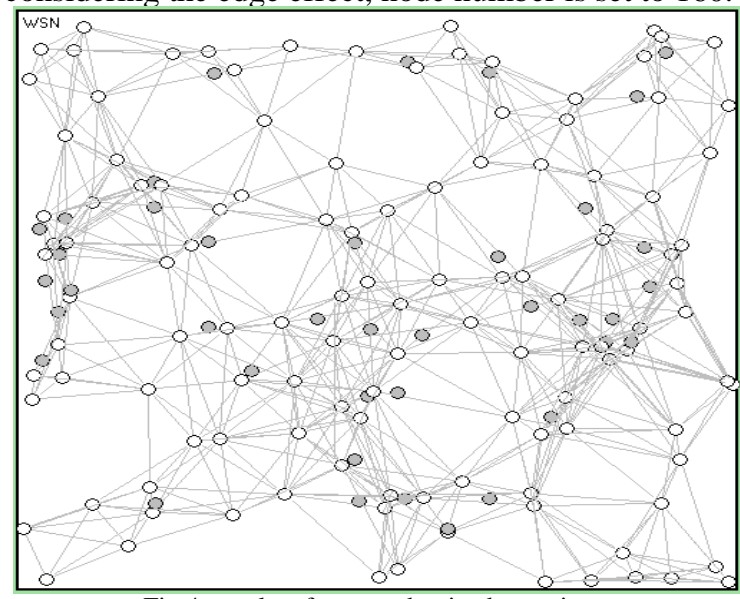

Fig.4 results of one randomized experiment

Figure 4 shows the partial results of one randomized experiment. Gray nodes represent dormant nodes, and white nodes are working nodes. It can be seen that nodes that have high coverage redundancy and belong to the independency set of nodes have been dormant. Table 2 shows the results of ten randomized experiments when node number $n$ is 160 and Preq $=99 \%$.

TABLE 2 EXPERIMENT RESULTS WHEN N IS 160 AND Preq $=99 \%$.

\begin{tabular}{|c|c|c|}
\hline Dormancy ratio & $\begin{array}{c}\text { Original } \\
\text { coverage ratio }\end{array}$ & $\begin{array}{c}\text { New coverage } \\
\text { ratio }\end{array}$ \\
\hline $25 \%$ & 0.989508 & 0.989404 \\
\hline $25.63 \%$ & 0.978292 & 0.978084 \\
\hline $22.50 \%$ & 0.993076 & 0.99248 \\
\hline $25 \%$ & 0.975352 & 0.975112 \\
\hline $25.63 \%$ & 0.972988 & 0.969744 \\
\hline $25 \%$ & 0.987872 & 0.987872 \\
\hline $25 \%$ & 0.974632 & 0.970628 \\
\hline $21.25 \%$ & 0.99544 & 0.995112 \\
\hline $26.88 \%$ & 0.980604 & 0.979716 \\
\hline $22.50 \%$ & 0.991044 & 0.991044 \\
\hline
\end{tabular}

The experimental results show that by executing PBRCA algorithm, the average value of coverage ratio variation is 0.0009612 , which is very little; while the average dormancy ratio reached $24.40 \%$. When the number of nodes is fixed to 160 and Preq is $90 \%$, ten randomized experiments results are shown in table 3 . The experimental results show 
that the average value of coverage ratio variation is 0.0087012 , while the average dormancy ratio reached $48.3 \%$, up to a maximum of $51.25 \%$.

TABLE 3 EXPERIMENT RESUlTS WHEN N IS 160 AND Preq $=90 \%$.

\begin{tabular}{|c|c|c|}
\hline Dormancy ratio & $\begin{array}{c}\text { Original } \\
\text { coverage ratio }\end{array}$ & $\begin{array}{c}\text { New coverage } \\
\text { ratio }\end{array}$ \\
\hline $45 \%$ & 0.991044 & 0.98664 \\
\hline $48.13 \%$ & 0.981316 & 0.97806 \\
\hline $49.38 \%$ & 0.98776 & 0.977504 \\
\hline $46.88 \%$ & 0.997876 & 0.993256 \\
\hline $46.88 \%$ & 0.997876 & 0.993256 \\
\hline $51.25 \%$ & 0.984816 & 0.967524 \\
\hline $48.75 \%$ & 0.989244 & 0.979348 \\
\hline $48.13 \%$ & 0.981948 & 0.97566 \\
\hline $49.38 \%$ & 0.991128 & 0.98372 \\
\hline $49.38 \%$ & 0.975496 & 0.956524 \\
\hline
\end{tabular}

Next, when the number of nodes is 80 and Preq $=99 \%$ the results of five randomized experiments are shown in table 4 .

TABLE 4 EXPERIMENT RESULTS WHEN N IS 80 AND Preq =99\%.

\begin{tabular}{|c|c|c|}
\hline $\begin{array}{c}\text { Dormancy } \\
\text { ratio }\end{array}$ & $\begin{array}{c}\text { Original } \\
\text { coverage ratio }\end{array}$ & $\begin{array}{c}\text { New coverage } \\
\text { ratio }\end{array}$ \\
\hline $6.25 \%$ & 0.913524 & 0.913524 \\
\hline $6.25 \%$ & 0.883068 & 0.883048 \\
\hline $3.75 \%$ & 0.903104 & 0.903104 \\
\hline $7.50 \%$ & 0.960104 & 0.960104 \\
\hline $5.00 \%$ & 0.858036 & 0.857612 \\
\hline
\end{tabular}

Experiments show that the average value of coverage ratio variation is just 0.000068 , while the average dormancy ratio reached $5.75 \%$, up to a maximum of $7.5 \%$.So when node number is less, the algorithm still works but its efficiency will decrease.

Above experimental results show that PBRCA could realize effective redundancy node control in WSN under different node density and coverage ratio at very low cost.

\section{CONCLUSION}

Aiming at the shortcomings of existing redundancy control schemes redundant node definition based on probability estimate is introduced in this paper for the scene of random uniform distribution and redundancy control algorithm PBRCA suitable for WSN are proposed. Theoretical analysis and experimental results show that PBRCA algorithm is adaptable and has high node dormancy ratio and little coverage ratio variation. In addition, computing complexity of PBRCA is lower than many known algorithms. However, PBRCA does not consider multiple order coverage. In fact, when network is unstable or node energy is low, some dormant redundant nodes can resume working state to improve network reliability and extend the lifetime of WSN [8].

\section{ACKNOWLEDGMENT}

This paper is supported by National Natural Science Foundation of China (NO: 61072043). This work is supported by NSFC (61072043)

\section{REFERENCES}

[1] Haitao Wang .Overview on Clustering Algorithm Wireless Sensor Networks[J]. The world of sensors,2011,4:6-10

[2] Lifeng Liu,Shihong Zou,Lei Zhang,Shiduan Cheng.Control algorithm of wireless sensor network density based on probability coverage model[J]. Journal of Beijing University of Posts and Telecommunications,2005,28(4):14-17.

[3] Gaojun Fan. Evaluation and improvement of wireless sensor network coverage performance[D].Changsha:National University of Defense Technology,2009

[4] Jiaqing Qu,Shu Zhang.Wireless sensor network lifetime optimization method of connectivity and coverage[J].Journal of Harbin Engineering University,2011,32(3):361-365.

[5] Aiming Wu.Research on wireless sensor network energy saving strategy based on CC2420[D].Chengdu:University of Electronic Science and technology,2008.

[6] Jie Chen,Xenofon Koutsoukos.Survey on Coverage Problems in Wireless Ad Hoc Sensor Networks[C].IEEE INFPCOM, 2001, 3 : 1380-1387.

[7] Shuguang Deng,Lianfeng Shen,Xinhui Chen,Xiaorong Zhu. Ondemand QoS protocol based on energy balance in large scale wireless sensor network[J].Journal of circuits and systems,2010,15(4):75-81.

[8] Jiaqing Qu.Research on wireless sensor network's lifetime[D].Harbin:Harbin Engineering University,2010. 